\title{
Lossless Feedback Amplifier Design
}

\author{
George D. Vendelin, Life Fellow, IEEE \\ Graduate Institute of Communications Engineering, Dept. of Electrical Engineering \\ National Taiwan University, Taipei, Taiwan
}

\begin{abstract}
Lossless feedback can be applied to three types of microwave amplifiers: (1) High Gain Amplifiers (HGA) Mason 1954 (2) Low Noise Amplifiers (LNA) Vendelin 1975 and (3) High Power Amplifiers (HPA). The LNA and HPA are duals of each other. This paper will give a tutorial review of all three feedback amplifiers.
\end{abstract}

Index Terms - Feedback, high gain amplifiers, low noise amplifiers, power amplifiers.

\section{INTRODUCTION}

The application of lossless feedback to microwave amplifiers has been well known for many years [1]-[2]. Beginning with Mason's classic paper [3], which was further reviewed by Gupta [4], the concept of unilateral gain was introduced in 1954, which was found to be invariant to common-lead and the highest stable gain achievable by the transistor. Later in 1975 Vendelin (and others) [5]-[7] showed low noise amplifiers could be improved by using lossless feedback, which was found to be normally a common-lead inductance in the source (emitter). The amplifier would give $F_{\min }$ and $\mathrm{S}_{11}=0$, a very useful improvement. Today we can add high power amplifiers may also be improved by using lossless feedback.

The properties of the three types of feedback amplifiers are summarized in Table 1 (after References). The Sparameters are $S_{11}=S_{22}=S_{12}=0$ and $S_{21}=\sqrt{U}<\theta$, where $U$ is defined in the next section.

\section{HIGH GAIN AMPLIFIERS}

Mason showed that using lossless feedback elements to reduce $Y_{12}$ or $S_{12}$ to zero would produce the unilateral gain when the input and output ports were simultaneously matched. This gain is given by:

$$
U=\frac{0.5 \cdot\left|S_{21} S_{12}-1\right|^{2}}{k \cdot\left|S_{21} S_{12}\right|-\operatorname{Re}\left(S_{21} S_{12}\right)}
$$

or equivalently by:

$$
U=\frac{\left|y_{21}-y_{12}\right|^{2}}{4 \cdot \operatorname{Re}\left(y_{11}\right) \operatorname{Re}\left(y_{22}\right)-4 \cdot \operatorname{Re}\left(y_{12}\right) \operatorname{Re}\left(y_{21}\right)}
$$

where $k$ is the well known stability factor, and the $y$ parameters are in the normalized form. The frequency where unilateral gain becomes unity is $f_{\max }$. By definition this represents the frequency range of activity for the device, i.e. where there is gain. Above $f_{\max }$ the transistor is passive. An oscillator can be built up to the $f_{\max }$, but no higher in theory, unless doubling is used [8]. It is interesting to note that Mason never published any unilateral amplifiers. Perhaps the reason is that stability at all frequencies is difficult to obtain when working with feedback networks; also the precision of the two-port parameters was probably poor and stability during the measurements would be difficult because of the lack of network analyzers.

Since extrapolating $U, G_{\mathrm{ma}}$ and $\left|S_{21}\right|^{2}$ to unity produces $f_{\max }, f_{\operatorname{mag}}$ and $f_{\mathrm{S} 21}$, one would normally expect

$$
\begin{gathered}
f_{\text {max }}>f_{\text {mag }}>f_{\mathrm{S} 21} \\
U>G_{\text {ma }}>\left|\mathrm{S}_{21}\right|^{2}
\end{gathered}
$$

but high frequency effects near $f_{\max }$ cause $f_{\max }=f_{\operatorname{mag}}$ [9]. In early transistors (Si BJT and GaAs MESFET) the shortcircuit gain, which is unity at $f_{\mathrm{T}}$, was always lower than $f_{\max }$. Today for the newer transistors, either $f_{\max }$ or $f_{\mathrm{T}}$ may be the higher number, based upon extrapolation of low frequency measurements.

\section{LOW NOISE AMPLIFIERS}

The noise figure of a transistor is given by:

$$
F=F_{\text {min }}+\frac{R_{n}}{G_{g}}\left|Y_{g}-Y_{o n}\right|^{2}
$$

where the minimum noise is achieved when $Y_{\mathrm{g}}=Y_{\mathrm{on}}$. Low noise amplifiers require an input matching network which provides the correct source or generator 
impedance $\left(Z_{\text {on }}=1 / Y_{\text {on }}\right)$ for minimum noise figure; this will normally result in $\left|S_{11}\right|>0$, which can be a problem in the overall system performance. Using a balanced amplifier structure, the input $S_{11}$ will approach zero, but this doubles the number of components. Also, by using a source (emitter) feedback inductor, the simultaneous noise and gain match may be achieved. The source inductance can resonate $C_{\mathrm{gs}}$ out in FET LNAs. It took about 5 years after the theory was published before designers picked up on this new design procedure for LNAs $[1,2,7]$.

\section{HIGH POWER AMPLIFIERS}

The performance of a power transistor may be characterized by an equation similar to (3) [1]-[2]:

$$
G_{L S}=G_{M A X}-\frac{R_{p}}{G_{L}}\left|Y_{L}-Y_{O P}\right|^{2}
$$

where the maximum large-signal gain and maximum output power are given when $Y_{\mathrm{L}}=Y_{\mathrm{op}}$. Several references are found in the literature [1]-[2] indicating power amplifiers may be improved by using feedback, but none were published which could achieve $S_{22}=0$ and the maximum Class A output power. It is useful to think of the HPA as the dual of the LNA when feedback is used. In the LNA, we move the $\Gamma_{\text {on }}$ to $S_{11}{ }^{*}$, so that an input match is also a noise match. In the same way for a HPA, we move $\Gamma_{\text {op }}$ to $S_{22}{ }^{*}$, so that an output match is also a power match. An example is recently demonstrated [10].

\section{CONCLUSION}

This paper has shown how lossless feedback may be used for HGA, LNA, and HPA to give a unilateral amplifier with the s-parameter matrix given by:

$$
S=\left[\begin{array}{ll}
0 & 0 \\
\sqrt{U} \angle \phi & 0
\end{array}\right]
$$

For the LNA, $F=F_{\min }$, and for the HPA $P_{1 \mathrm{dBc}}=P_{1 \mathrm{dBcmax}}$. The design for HGA and LNA have been known for over
30 years, but the design of the HPA is new and is just now being demonstrated [10].

\section{ACKNOWLEDGEMENT}

This paper has benefited from numerous discussions with Calvin Chien and Luke Hsu Sun Huang of Santa Clara University (CA) and Zou-Min Tsai, Ming-Fong Lei, Kuo-Jung Sun, Chin-Shin Lin, and Pin-Cheng Huang of National Taiwan University. It was also edited by Professor Huei Wang of National Taiwan University.

\section{REFERENCES}

[1] G. D. Vendelin, A. M. Pavio, and U. L. Rohde, Microwave Circuit Design Using Linear and Nonlinear Techniques, Wiley, 1990.

[2] G. D. Vendelin, A. M. Pavio, and U. L. Rohde, Microwave Circuit Design Using Linear and Nonlinear Techniques, $2^{\text {nd }}$ Ed. , Wiley, 2005.

[3] S. J. Mason, "Power Gain in Feedback Amplifiers," IRE Trans. on Circuit Theory, vol. CT-1, June 1954, pp. 20-25.

[4] M. S. Gupta, "Power Gain in Feedback Amplifiers, a Classic Revisited," IEEE Trans. Microwave Theory and Tech., vol. 40, May 1992, pp. 864-879.

[5] G. D. Vendelin "Feedback Effects on the Noise Performance of GaAs MESFETs," IEEE Microwave Symposium Digest, 1975, pp. 324-326.

[6] L. Besser, "Stability Considerations of Low-Noise Transistor Amplifiers with Simultaneous Noise and Power Match," IEEE Microwave Symposium Digest, 1975, pp. 327329.

[7] M. P. van der Heijden, L. C. N. Vreede, and J. N. Burghartz, "On the design of unilateral dual-loop feedback low-noise amplifiers with simultaneous noise, impedance, and IIP3 match," IEEE Journal of Solid-State Circuits, vol. 39, Oct. 2004, pp. 1727-1736.

[8] P. C. Huang, M. D. Tsai, H. Wang, C. H. Chen, and C. S. Chang, "A 114-GHz VCO in 0.13 um CMOS Technology," IEEE International Solid-State Circuits Conference Digest, Feb 2005, pp. 404-405.

[9] Michael B. Steer and Robert J. Trew, "High-frequency limits of millimeter-wave transistors," IEEE Electron Device Letters, vol. EDL-7, no. 11, Nov. 1986, pp. 640-642.

[10] Z. M. Tsai, K. J. Sun, H. Wang, and G. D. Vendelin, "Unilateralization of Power Amplifiers with Simultaneous Output Power and Impedance Match by Lossless Feedback," submitted for publication to IEEE Trans. Microwave Theory and Tech., 2005.

Table 1: Summary of Feedback Amplifier Properties

\begin{tabular}{|l|l|l|}
\hline High Gain Amplifier (HGA) & Low Noise Amplifier (LNA) & High Power Amplifier (HPA) \\
\hline$\Gamma_{\mathrm{G}}=\mathrm{S}_{11}{ }^{*}$ & $\Gamma_{\mathrm{G}}=\Gamma_{\mathrm{on}}$ & $\Gamma_{\mathrm{L}}=\Gamma_{\mathrm{op}}$ \\
\hline$\Gamma_{\mathrm{L}}=\mathrm{S}_{22}{ }^{*}$ & $\mathrm{~F}=\mathrm{F}_{\min }$ & $\mathrm{P}_{\mathrm{IdBc}}=\mathrm{P}_{1 \mathrm{dBcmax}}$ \\
\hline
\end{tabular}

